\title{
Prophylactic use of fluconazole in neutropenic cancer patients
}

\author{
Kadri Yamaç, Esin Şenol, Rauf Haznedar
}

\begin{abstract}
Summary
This study was carried out on 70 patients with haematological or solid malignancies who were receiving chemotherapy and/or radiotherapy. Forty-one patients were randomly assigned to receive fluconazole, $400 \mathrm{mg} / \mathrm{day}$, while they were neutropenic. Systemic fungal infection developed in four of the 41 patients $(9 \%)$ receiving prophylaxis in comparison to nine of 29 patients (31\%) not receiving prophylaxis. The incidence of systemic fungal infection was significantly different between the groups receiving prophylaxis and those not receiving it $(p<$ $0.05)$. Fluconazole was found to be effective for preventing systemic fungal infections in neutropenic patients with cancer.
\end{abstract}

Keywords: fluconazole, prophylaxis, fungal infection

\section{Introduction}

Fungal infections are becoming an increasing problem in cancer patients and factors that play an important role in this increase include difficulties in establishing the diagnosis and limitations of management. Although amphotericin B is the agent of choice for the treatment of systemic fungal infections, its use is limited by considerable toxicity. ${ }^{1}$ These factors and the high mortality rate associated with fungal infections have made prophylaxis more important. ${ }^{2}$ Until now, prophylaxis with nystatin, amphotericin B, clotrimazole, ketaconazole, and miconazole has been tried but found to be unsatisfactory. ${ }^{3,4}$

Fluconazole is a bistriazole antifungal agent, which is safe, well-tolerated and highly active against most of the pathogens that cause systemic fungal infections. ${ }^{5,6}$ Studies suggest that fluconazole would be effective in neutropenic hosts. ${ }^{3,7}$ This study was conducted to determine the efficacy of prophylaxis with fluconazole in neutropenic cancer patients.

\section{Methods}

Of the 70 neutropenic cancer patients enrolled in the study, 20 were female and 50 male. Their ages were 16 to 68 (mean $45 \pm 15$, median 49.5) years. The distributions of the primary tumour are shown in the table. Patients were defined as neutropenic when the neutrophil count was below $2000 / \mu 1$. The mean neutrophil counts were $822 \pm 645 / \mu \mathrm{l}$ (range $0-1908 / \mu \mathrm{l}$ ).

Patients were randomly assigned to receive or not to receive prophylaxis with fluconazole. Prophylaxis was given to 41 patients; 29 patients did not receive any prophylaxis. Fluconazole was given orally in a dose of $400 \mathrm{mg}$ daily in two divided doses. Treatment was continued until the neutrophil count had increased to $2000 / \mu$ l or until systemic fungal infection was suspected. During the treatment period cultures of throat, feces, urine and blood were obtained twice weekly. In addition, patients were evaluated for signs and symptoms of infection. Systemic fungal infection was considered to be present when the organism was isolated from blood or urine and when yeasts and pseudohyphae were microscopically seen on sterile urine smears.

\section{STATISTICAL ANALYSIS}

Student's unpaired $t$-test was used for comparison of variables, eg, absolute neutrophil counts. Nominal results, eg, incidence of Candida infection, between groups were compared using the chi-squared test. A p-value below 0.05 was considered significant. Data are reported as mean \pm standard deviation (SD).

\section{Results}

Systemic fungal infection occurred in four of 41 patients $(9.8 \%)$ who were given prophylaxis with fluconazole and in nine of 29 patients $(31 \%)$ who were not $(p<0.05)$.

The absolute neutrophil counts, in the fluconazole group were $763 \pm 91 / \mu$ l for cases

Table Patient characteristics

\begin{tabular}{lll}
\hline Patient characteristics & Control & Fluconazole \\
\hline Number (n) & 29 & 41 \\
Median age (range) (years) & 50 & 49 \\
& $(16-67)$ & $(17-68)$ \\
Sex & & \\
male & 20 & 30 \\
female & 9 & 11 \\
Underlying disease & & \\
acute leukemia & 10 & 13 \\
chronic leukemia & 3 & 2 \\
Hodgkin's disease & 4 & 5 \\
non-Hodgkin's lymphoma & 5 & 9 \\
myelodysplastic syndrome & 2 & 3 \\
aplastic anemia & - & 2 \\
solid tumour & 5 & 4 \\
multiple myeloma & - & 3 \\
\hline
\end{tabular}

\author{
Dr Kadri Yamaç, Seny \\ Ankara, Turkey \\ Accepted 2 February 1995 \\ Department of
Hematology, Gaz \\ University, Faculty of \\ Turkey \\ Department of \\ Gazi University, \\ Ankara, Turkey \\ E Senol \\ Correspondence to
}


with systemic fungal infection and $739 \pm 610 /$ $\mu l$ for cases without $(\mathrm{p}>0.05)$, while among the controls, the mean neutrophil counts for cases with systemic fungal infection were $949 \pm 727$ / $\mu \mathrm{l}$ and $898 \pm 704 / \mu \mathrm{l}$ for cases without ( $>0.05$ ). Patients with and without systemic fungal infection were also compared for the duration of neutropenia. The mean duration of neutropenia was $25 \pm 17$ days for the fluconazole patients and $17 \pm 16$ for the controls; the difference was significant $(p<0.05)$.

A breakdown of Candida infections in different groups of patients according to the underlying disease revealed that eight of the 13 Candida-positive cases were acute leukemia $61.5 \%$ (the other cases were one each of nonHodgkin's lymphoma, Hodgkin's disease, aplastic anemia, solid tumour and chronic leukemia).

\section{Discussion}

Invasive fungal infections are important causes of morbidity and mortality among cancer patients. Candida and Aspergillus are the most common causes of invasive fungal infections and Candida species predominate in cancer patients. ${ }^{3,8,9}$ Several autopsy studies have shown that $10-30 \%$ of patients with leukemia $10-15 \%$ of patient with lymphoma and $5 \%$ of patients with solid tumours have invasive candidiasis. ${ }^{4}$ In another report the incidence of systemic Candida infection in immunocompromised patients was $37.5 \%$ on autopsy. ${ }^{10}$ In our study the $31 \%$ incidence of systemic fungal infections noted among the patients receiving no prophylaxis, with predominantly acute leukemia as an underlying disease, is parallel to reports in previous studies. ${ }^{4}$ The results of this study are also similar to previous reports on the significance of the duration of neutropenia as a pre-disposing factor for fungal infections. ${ }^{11}$

Difficulties in diagnosis and management are the major problems encountered in fungal infections. Diagnosis is established by demonstration of tissue invasion histologically or by isolating the fungus from urine or blood. ${ }^{4}$ However, in high-risk patients, isolating the fungus is difficult and blood cultures lack sensitivity. ${ }^{12}$ It has been shown that blood cultures of patients with autopsy-proven fungal infection were $50 \%$ negative. ${ }^{13}$ In this study, fungus could not be isolated from blood cultures of cases diagnosed as having systemic fungal infection.

According to an autopsy study of systemic Candida infections, the kidney is the most frequently involved organ and candiduri may be the first manifestation of systemic infection. $^{14}$ In particular, the appearance of pseudohyphae is indicative of invasive Candida infection rather than colonisation. ${ }^{15}$ In our study patients with microscopical appearance of yeast and pseudohyphae in urine specimens were considered to have systemic Candida infection. Although accurate diagnosis of candidiasis relies on histological demonstration, in these patients the use of such invasive procedures is impossible for several reasons. ${ }^{16}$

\section{Learning/summary points}

- fungal infections are frequent among neutropenic cancer patients

- fluconazole is a highly effective and well-tolerated prophylactic agent in neutropenic patients with cancer

Several problems are encountered in the management of fungal infections, such as establishing a definite diagnosis at an early stage and the high toxicity of antifungal agents. As antifungal agents are initiated empirically, subjects without fungal infection are unnecessarily exposed to drug toxicity. On the other hand, some patients with fungal infection are not given any antifungal therapy or are given it too late. While amphotericin B is the drug of choice for the treatment of systemic fungal infections, it is not always effective, must be given parenterally, and is associated with high toxicity. ${ }^{17}$ In several reports failure of amphotericin B has been noted in $40-50 \%$ of cases. ${ }^{6,9}$

The difficulty in establishing the diagnosis, problems with management and the high mortality rates associated with this disease makes prophylactic therapy highly desirable. However, agents for prophylactic use must be effective, safe and well-tolerated.

Fluconazole is a well-tolerated agent which is active against many important fungal pathogens which cause systemic fungal infections in cancer patients. It has a long half-life which allows once-daily administration via either oral or intravenous routes. ${ }^{7,8}$ Experimental studies in granulocytopenic animals have indicated that fluconazole is effective for preventive treatment of systemic Candida infection, penetrates well into all tissue sites, has a low toxicity and high doses are effective in prophylaxis against disseminated aspergillosis. ${ }^{3}$ Clinical studies have shown that prophylaxis with fluconazole reduced the incidence of both superficial and systemic Candida infections, but the incidence of colonisation by strains other than $C$ albicans (eg, $C$ kruzei and Torulopsis glabrata) have increased. ${ }^{2,9,16,18-20}$ Although the Candida species were not identified in the present study, it is probable that the systemic infections observed in some of the fluconazole recipients, could be due to fluconazole-resistant non-albicans strains. Prophylaxis with fluconazole is associated with the emergence of less virulent Candida species but offers the advantage of suppression of more virulent Candida species such as $C$ albicans.

In this study the dose of fluconazole was relatively high $(400 \mathrm{mg} /$ day $)$ as we hoped that this dose might also offer some protection against Aspergillus.

Our findings support the use of highly effective prophylactic agents like fluconazole in neutropenic cancer patients. Studies are still needed to establish more rapid diagnostic methods for systemic fungal infections and to develop more effective, less toxic therapies. 
1 Walsh TJ, Lee J, Aoki S, et al. Experimental basis for use of fluconazole for preventive or early treatment of disseminat candidiasis in granulocytopenic hosts. Rev Infect Dis 1990, 12 (suppl 3): 307-17.

2 Inci $R$, Tümay E. Antifungal chemotherapy in antimicrobic chemotherapy, In: Meco O, Willke A, Balik I, Kurt H. Türk Microbiol Soc Rep 1992, 16-35.

3 Milliken ST, Powles RL. Antifungal prophylaxis in bone marrow transplantation. Rev Infect Dis 1990; 12 (suppl 3): 373-9.

4 Meunier F. Candidiasis. Eur $\mathcal{f}$ Clin Microbiol Infect Dis 1989; 8: 438-47.

5 Sugar AM. Empiric treatment of fungal infections in the neutropenic host. Reviews of the literature and guidelines neutropenic host. Reviews of the literature and
for use. Arch Intern Med 1990; 150: 2258-64.

6 Troke PF, Andrews RJ, Pye GW, Richardson K Fluconazole and other azoles, Translation of in vitro activity to in vivo and clinical efficacy. Rev Infect Dis 1990; 12 (suppl 3): $276-80$

7 Anaissie E. Opportunistic mycoses in immunocompromised host. Experience at a cancer center and review. Clin Infect Dis 1992; 14 (suppl 1): 43-53.

8 Walsh TJ, Lee J, Lecciones J, et al. Empiric therapy with amphotericin B in febrile granulocytopenic patients. Rev Infect Dis 1991; 13: 496-503.

9 Richet HM, Andramont A, Tancrede C, Pico LJ, Jarvis WR. Risk factors for candidemia in patients with acute lymphocytic leukemia. Rev Infect Dis 1991; 13: 211-5.

10 Meunier F, Aoun M, Bitar N. Candidemia in immunocompromised patients. Clin Infect Dis 1992; 14 (suppl 1): compro.
11 Bodey PG. Infections in cancer patients. Am $\mathcal{F}$ Med 1986; 81 (suppl 1A), 11-26.

12 Perfect JR. Fungal infections. Curr Opin Infect Dis 1992; 5 433-6.

13 Bennet JE. Mycoses. In: Mandell, Douglas, Bennet (eds) Principles and practice of infectious disease, 3 rd edn. Churchil Livingstone, 1990; pp 1943-58.

14 Crislip MA, Edwards JE. Candidiasis in systemic fungal infections. Infect Dis Clin N Am 1989; 3: 100-33.

15 Öztop F. Pathology of Candida infections. Turk Microbiol Soc Rep 1986; 6: 41-5.

16 Saral R. Candida and Aspergillus infections in immunocompromised patients: an overview, Rev Infect Dis 1991; 13: 487-92.

Graybill JR. New anti fungal agents. Eur $\mathcal{f}$ Clin Microbio Infect Dis 1989; 8: 402-12.

18 Waran RE. Prevention of infection. Curr Opin Infect Dis 1992; 5: 409-14.

19 Goodman JL, Winston DJ, Greenfield RA, et al. A controlled trial of fluconazole to prevent fungal infections in patients undergoing bone marrow transplantation. $N$ Engl $\mathcal{F}$ Med 1991; 326: 845-51.

20 Drew JW, Pranatharthi HC, Hilliard ML, et al. Fluconazole prophylaxis of fungal infections in patients with acute eukemia, results of randomized placebo-controlled, double blind multicenter trial. Ann Intern Med 1993; 118: 495-503. 